\title{
La Precariedad Laboral y Calidad de Vida de Grupos Generacionales en Organizaciones de Puerto Rico durante Trabajo Remoto
}

\author{
The Precariousness of Work and Quality of Life of General Groups in \\ Organizations of Puerto Rico during Remote Work
}

\author{
Keila Edith Oyola Rosa ${ }^{{ }^{*}}$, Antonio Zapata Solá ${ }^{2}$, \& Ángela M. Cruz Félix ${ }^{3}$ \\ 1 Universidad Albizu, Recinto de San Juan, Puerto Rico. (D) https://orcid.org/0000-0002-5773-940X \\ 2 Universidad Albizu, Recinto de San Juan, Puerto Rico. (D) https://orcid.org/0000-0003-2742-2732 \\ 3 Universidad Albizu, Recinto de San Juan, Puerto Rico. (D) https://orcid.org/0000-0002-1989-285X \\ * Correspondencia: koyola@albizu.edu
}

Recibido: 15 junio 2021 | Aceptado: 30 julio 2021 | Publicado: 24 diciembre 2021

WWW.REVISTACARIBENADEPSICOLOGIA.COM

\section{Citar como:}

Oyola Rosa, K. E., Zapata Solá, A., \& Cruz Félix, A. M. (2021). La precariedad laboral y calidad de vida de grupos generacionales en organizaciones de Puerto Rico durante trabajo remoto. Revista Caribeña de Psicología, 5, e5623. https://doi.org/10.37226/rcp.v5i1.5623

\section{RESUMEN}

El objetivo de esta investigación fue construir y validar una escala para medir la percepción de la calidad de vida laboral durante el trabajo remoto y los diferentes factores que la impactan según la brecha generacional. En esta investigación se desarrolló una escala validada con una muestra de 210 participantes del ambiente laboral y residentes en Puerto Rico. Donde 62\% reportaron ser del género femenino $(62 \%, n=131)$, y el 37\% reportaron ser del género masculino $(37 \%, n=77)$. Se realizaron los siguientes análisis: propiedades psicométricas entre éstos: análisis factorial exploratorio, Axis Factoring y rotación Oblimin, validez de contenido, análisis de confiabilidad con coeficiente alfa de Cronbach y para consistencia interna. A través del análisis de los resultados, se concluyó que la escala tiene propiedades psicométricas es útil para lo que fue diseñada, ya que obtuvo un alfa de Cronbach aceptable ( $\alpha$ $=.72$ ). El construir una escala que mida calidad de vida laboral durante el trabajo remoto contribuyó al diseño de un nuevo instrumento y como base para investigaciones futuras.

Palabras Claves: precariedad laboral, calidad de vida, calidad de vida laboral, grupo generacional

\begin{abstract}
The objective of this research was to build and validate a scale to measure the perception of the quality of work life during remote work and the different factors that impact it according to the generation gap. In this research, a validated scale was developed with a sample of 210 participants from the work environment and residents of Puerto Rico. Where $62 \%$ reported being female $(62 \%, n=131)$, and $37 \%$ reported being male $(37 \%, n=77)$. The following analyzes were carried out: psychometric properties between systems: exploratory factor analysis, Oblimin axis and rotation factor, content validity, reliability analysis with Cronbach's alpha coefficient and for internal
\end{abstract}


consistency. Through the analysis of the results, it was concluded that the scale has psychometric properties and is useful for what it was designed for, since it obtained an acceptable Cronbach's alpha $(\alpha=.72)$. Building a scale that measures quality of working life during remote work contributed to the design of a new instrument and as a basis for future research.

Keywords: labor precariousness, quality of life, quality of labor life, generational group

\section{INTRODUCCIÓN}

El ámbito laboral ocupa una buena parte del tiempo de las personas y tiene fuertes implicaciones para la salud, el bienestar, la cohesión social y desarrollo personal o por el contrario alimentación, malestar y enfermedad (Moreno \& Camino, 2019). Por lo que es importante considerar las implicaciones que atentan contra la seguridad y salud en este espacio de desarrollo (Moreno \& Camino, 2019). Martínez (2017) define la precariedad laboral como las condiciones de trabajo en que se vincula el trabajador y como este desempeña sus tareas y funciones en base a un estado de inseguridad. Acorde con esto, Marrero y García (2018), establecen que la precariedad laboral es un constructo que incluye la economía y la inseguridad laboral que repercute en la salud física como mental. La precariedad laboral es una problemática que está afectando al país y al mundo través de los años (Marrero \& García, 2018). Según Arias y Ordoñez (2018), el empleo precario es un constructo multidimensional que incluye aspectos como la inseguridad laboral, el nivel de salario bajo, la privación económica y una protección social limitada, así como un bajo ejercicio de los derechos laborales.

\section{Precariedad Laboral}

A su vez, Díaz (2019), indica que la incertidumbre económica, el rápido crecimiento del sector de servicios, la intensa competencia mundial, la feminización de la fuerza de trabajo y los avances tecnológicos de las últimas décadas atribuyen a la precariedad laboral. También, se le atribuye a los vacíos o zonas grises en las leyes reguladoras del empleo, fueron fuerzas que impulsaron el alza en el empleo atípico (Díaz, 2019). En consecuencia, y como medida para posibilitar la prevención de la precariedad laboral en Puerto Rico se puede trabajar mediante la mejora de las condiciones de trabajo. Las mismas deben estar sujetas a condiciones justas para todos los empleados (Marrero \& García, 2018). Así, como priorizar las necesidades del empleado para que éstos puedan ejercer sus funciones de forma efectiva y apropiada (Marrero \& García, 2018). Es importante puntualizar que la economía ha cambiado a nivel mundial y esto ha provocado la escasez laboral. Por consiguiente, esta repercute en la inestabilidad laboral para los trabajadores (Díaz, 2019).

Referente a la modalidad de trabajo remoto, esta práctica surge en los años 70 durante la crisis petrolera en Estados Unidos. Los empleados presentaban dificultad para desplazarse a sus lugares de trabajo (Peralta-Beltrán et al., 2020). En la actualidad esta práctica ha incrementado y más con los avances tecnológicos que han implementado cambios drásticos en los modelos de operación y organización de las industrias, así como el desempeño del recurso humano (Peralta-Beltrán et al., 2020). Unos de estos cambios ha sido el experimentar y ejercer las funciones laborales remotamente por las emergencias y avances tecnológicos (Fernández, 2017). Estos avances tecnológicos han desarrollado herramientas para ejercer diferentes empleos, lo que ha incrementado la desaparición de profesiones tradicionales, siendo estas reemplazadas y provocando la reducción de oportunidades de empleo (Fernández, 2017). Hoy día, como parte de la actual crisis por la pandemia del COVID19, la mayoría de las industrias optaron por adaptarse al trabajo remoto completamente para continuar brindando sus servicios (Peralta-Beltrán et al., 2020).

\section{Calidad de Vida}

El concepto de calidad de vida se define desde la perspectiva de varios autores que coinciden en que este es uno multidisciplinario, ya que se puede utilizar en diferentes contextos. El concepto calidad de vida ha sido utilizado en diversos ámbitos tales como la psicología, la salud, la educación, la economía y la política. Esto a promovido el interés sobre el constructo a diferentes niveles de generalización (Benítez, 2016). A su vez, la calidad de vida fue circulando por diferentes estadios cuyos indicadores se relacionaban directamente con la percepción que tienen los seres 
humanos respecto a su vida, a sus derechos y todo lo que beneficie su existencia (Romina, 2016). De igual forma, la calidad de vida relaciona diferentes niveles de estudio que conducen a abordar un sentido amplio del término (Custodio et al., 2017).

La calidad de vida en el ámbito laboral se refiere a la satisfacción de los trabajadores respecto a sus necesidades. Estas necesidades están relacionadas con estabilidad laboral, equilibrio entre su familia, motivación, seguridad y así como obtener un salario justo y equivalente a sus funciones (Castro et al., 2018). A su vez, Cruz (2018) lo define como la percepción de los empleados sobre su grado de satisfacción e insatisfacción de la organización en la cual laboran y como esta resulta en una evaluación del grado de bienestar y el desarrollo que estas condiciones generan en los recursos humanos. De igual forma, se ha identificado que la calidad de vida guarda relación con las condiciones laborales, ya que éstas son cualquier característica que pueda influir en los riesgos para la seguridad y salud del trabajador (Castro et al., 2018). En este caso, el riesgo laboral es cuando un empleado pudiera sufrir algún daño a causa de su trabajo. Entre los riesgos laborales que se han identificado está el estrés laboral, que se puede manifestar con diferentes síntomas como lo son: cansancio, desánimo, ansiedad, pérdida de ingresos y desempleo.

\section{Grupos Generacionales}

Las generaciones han sido objeto de estudios por décadas debido a los diferentes cambios que han experimentado en varios aspectos, pero se acentúa más en el ámbito laboral (Díaz et al., 2017). Existen cinco generaciones, la Generación Tradicionalistas (19151925), Generación Silenciosa (1926-1945), Generación “Baby Boomers" (1946-1960), Generación X (19611981), Generación “Millenials" o Y (1982-2000) y Generación Z o “Centennials" (2001 hasta la fecha) (Díaz et al., 2017). Sin embargo, las generaciones que actualmente contribuyen al ámbito laboral son tres: los "Baby Boomers", Generación X, Generación "Millennials" o Y (Díaz et al., 2017).

Es importante mencionar que las diferencias entre las generaciones causan cambios significativos con relación a las actitudes, los pensamientos y los comportamientos que una persona presente en su carrera profesional, así como su interés por este. Por consiguiente, cada individuo adquiere nuevas expecta- tivas sobre las dimensiones de la vida a través de cada etapa de desarrollo, que también está influenciado por el contexto histórico y sociocultural creando unos denominadores que identifican a las personas según su época de nacimiento (Del Valle, et al., 2019). En este caso, cuando un "conjunto de personas que, habiendo nacido en fechas próximas y recibido educación e influjos culturales y sociales semejantes, adoptan una actitud en cierto modo común en el ámbito del pensamiento o de la creación" se le conoce como generación (Real Academia Española, 2019).

\section{Propósito de Investigación}

El propósito de esta investigación fue presentar cómo en las brechas generacionales se ve afectada su calidad de vida por la precariedad laboral durante el trabajo remoto. Así como desarrollar una escala para evaluar diferentes aspectos tales como: bienestar, salud, alimentación balanceada, entorno social y cultural, logros profesionales y trabajo remoto. Por ende, se formularon las siguientes preguntas de investigación: ¿Cuál es el efecto de la precariedad laboral en la calidad de vida según las brechas generacionales? ¿Cuál es el rol de la precariedad laboral ante la calidad de vida? ¿La Escala de percepción de calidad de vida laboral durante el trabajo remoto de los empelados en Puerto Rico cumple con las propiedades psicométricas adecuada para ser utilizada en investigaciones?

De igual forma, se establecieron objetivos para validar la información recopilada de la investigación, éstos fueron: Comprender la forma en que la precariedad laboral afecta la calidad de vida en las brechas generacionales en los trabajadores en las organizaciones. Relacionar la precariedad laboral de los trabajadores de las organizaciones con las brechas generacionales. Construir y validar una escala para medir la percepción de la calidad de vida laboral durante el trabajo remoto y los diferentes factores que la impactan según las brechas generacional. Diferenciar los resultados de la escala considerando la información sociodemográfica de la muestra.

\section{MÉTODO}

\section{Diseño de Investigación}

El diseño que se utilizó fue transversal instrumental (Ato et al., 2013) con metodología descriptiva de un grupo con el propósito de elaborar, aplicar y 
validar un instrumento construido para evaluar la calidad vida laboral durante el trabajo remoto.

\section{Participantes}

Los participantes de esta investigación fueron 210 personas mayores de edad, donde $37 \%$ son del género masculino y $62 \%$ del género femenino, éstos tenían como criterios de inclusión tener más de 21 años, estar trabajando haber trabajado de forma remota, saber leer y escribir en español y vivir en Puerto Rico (ver Tabla 1).

Tabla 1

Datos Sociodemográficos $(n=210)$

\begin{tabular}{|c|c|c|}
\hline & $F$ & $\%$ \\
\hline \multicolumn{3}{|l|}{ Género } \\
\hline Femenino & 131 & 62.38 \\
\hline Masculino & 79 & 37.62 \\
\hline \multicolumn{3}{|l|}{ Edad } \\
\hline $21-39$ años & 105 & 50.00 \\
\hline $40-60$ años & 82 & 39.05 \\
\hline $61-75$ años & 23 & 10.95 \\
\hline \multicolumn{3}{|l|}{ Preparación Académica } \\
\hline Escuela Superior & 3 & 1.43 \\
\hline Cursos Técnico & 5 & 2.38 \\
\hline Cursos Universitarios & 27 & 12.86 \\
\hline Bachillerato & 97 & 46.19 \\
\hline Maestría & 64 & 30.48 \\
\hline Doctorado & 14 & 6.67 \\
\hline \multicolumn{3}{|l|}{ Estado Civil } \\
\hline Soltero(a) & 46 & 21.90 \\
\hline Casado(a) & 77 & 36.67 \\
\hline Convive & 52 & 24.76 \\
\hline Divorciado(a) & 17 & 8.10 \\
\hline Con pareja & 18 & 8.57 \\
\hline \multicolumn{3}{|l|}{ Estatus Laboral } \\
\hline Tiempo Completo & 155 & 73.81 \\
\hline Tiempo Parcial & 18 & 8.57 \\
\hline Por Contrato & 34 & 16.19 \\
\hline Desempleado(a) & 3 & 1.43 \\
\hline \multicolumn{3}{|l|}{ Lugar de Empleo } \\
\hline Gobierno & 39 & 18.57 \\
\hline Privado & 143 & 68.10 \\
\hline ONG, Sin fines de lucro & 17 & 8.10 \\
\hline Federal & 2 & 0.95 \\
\hline Cuenta Propia & 9 & 4.29 \\
\hline \multicolumn{3}{|l|}{ Tiempo en la Organización } \\
\hline Menos de 1 año & 19 & 9.05 \\
\hline 1 año a 3 años & 28 & 13.33 \\
\hline 4 años a 6 años & 60 & 28.57 \\
\hline 7 años a 9 años & 53 & 25.24 \\
\hline 10 años o más & 50 & 23.81 \\
\hline
\end{tabular}

\section{Instrumentos}

Se utilizaron dos instrumentos. En primer lugar, un instrumento para recopilar los datos sociodemográficos de los participantes como género, edad, puesto que ocupa, nivel de escolaridad, lugar de empleo y tiempo en la organización. Así como si actualmente residían en Puerto Rico, y si éstos contaban con calidad de vida laboral e identificar en los estilos de hacer tareas laborales respecto a los empleados mayores o más jóvenes.

El segundo instrumento fue la escala La Calidad de Vida Laboral durante el Trabajo Remoto, constaba de 34 reactivos y se contestaba en escala Likert que va del 1 al 5 desde "Totalmente en Desacuerdo" hasta "Totalmente de Acuerdo". Contiene seis dimensiones las cuales son: (a) Bienestar, (b) Salud, (c) Alimentación Balanceada, (d) Entorno Social y Cultural, (e) Logros Profesionales y (f) Trabajo Remoto. La confiabilidad del mismo fue de .72 con la técnica de Alpha de Cronbach.

\section{Procedimientos Generales}

La investigación fue aprobada por el Comité de Ética (IRB por sus siglas en inglés) de la Universidad Albizu. Una vez aprobada, se ingresaron los cuestionarios en la plataforma electrónica PsychData. Una vez los participantes accedían al enlace provisto para la investigación, leían detenidamente la hoja de consentimiento y de estar de acuerdo a participar, procedían a acceder los cuestionarios. En el consentimiento informado se les informó sobre su participación voluntaria, la confidencialidad de su participación y el acceso a la información de la investigación. El lenguaje utilizado en el consentimiento informado fue una claro y preciso para la comprensión del lector.

Además, se les explicó a los participantes el propósito de la investigación. Así mismo se les garantizó que al terminar la investigación la información se almacenará en archivos computarizados y se garantiza la confidencialidad y anonimato. De igual forma, para garantizar los derechos y la seguridad física y emocional de los participantes, se desarrolló un protocolo para eventos adversos no esperados. Por último, se les notificó a los participantes que de necesitar información sobre la investigación se podrá comunicar con la investigadora principal del estudio. 


\section{Análisis de Datos}

Para realizar los análisis de esta investigación se utilizó el programa estadístico IBM SPSS Statistics (versión 27). Se realizaron análisis de propiedades psicométricas para la escala de calidad de vida desarrollada para esta investigación. Entre los análisis de propiedades psicométricas se realizaron análisis de factores exploratorio con una extracción Principal Axis Factoring y una rotación Oblimin. Como criterio de eliminación de reactivos para el análisis de factores exploratorio se consideraron cargas factoriales menores de .30 o reactivos que compartieran carga factorial con otro factor.

Para examinar la adecuacidad de la muestra en el análisis factorial, se utilizaron los criterios de la prueba de Kaiser-Meyer-Olkin (KMO) y la esfericidad de Bartlett. Además, se calcularon los índices de discriminación para los factores encontrados, así como la consistencia interna de estos mediante el coeficiente alfa de Cronbach. En adición, se realizaron análisis de correlación entre los factores de la escala de calidad de vida, y entre algunas variables sociodemográficas.

Además, se realizó un análisis de normalidad Shapiro-Wilk para los factores de la escala por las variables sociodemográficas y se encontró que no hay una distribución normal de los datos para ninguno de los factores por los datos sociodemográficos. Ante esto, se realizaron análisis de comparación grupal no paramétricos (U de Mann-Whitney y Kruskal-Wallis) para examinar si existen diferencias entre las puntuaciones de los factores por las variables sociodemográfica.

\section{RESULTADOS}

Para estudiar la estructura factorial del instrumento de calidad de vida desarrollado se realizó un análisis de factores exploratorio. En el análisis se incluyeron todos los reactivos del instrumento (34 reactivos). El análisis pasó por siete fases en total. En la primera fase se cumplió con la adecuacidad de la muestra $\left(K M O=.85, \chi^{2}(561)=3,645.60\right)$. Se detectaron ocho factores que explicaban el $65.59 \%$ de la varianza. Sin embargo, se tuvieron que eliminar reactivos porque compartían carga factorial (E6, T2, S3, T3, L2). En la segunda fase se observó que se mantuvo la adecuacidad de la muestra $\left(K M O=.84, \chi^{2}(406)=2,835.54\right)$. Se mantuvieron de igual forma ocho factores explicando el $67.56 \%$ de la varianza. De forma similar a la fase uno, se eliminó un reactivo porque compartía carga factorial con otro factor (L1). En la tercera fase, se mantuvo la adecuacidad de la muestra $(K M O=.84$, $\left.\chi^{2}(378)=2,733.25\right)$. En esta ocasión se observaron siete factores que explicaban un $65.07 \%$ de la varianza, y se eliminaron dos reactivos que compartían carga factorial con otros factores (S5, A3) y dos reactivos que no cumplieron con el mínimo de carga factorial (E4, E2).

En la cuarta fase, la adecuacidad de la muestra mantuvo sus valores dentro de lo esperado $(K M O=$ $\left..82, \chi^{2}(276)=2,165.34\right)$. En esta fase se observaron siete factores que explicaban un $68.31 \%$ de la varianza. Como se observaron reactivos con carga factorial negativa se procedieron a eliminar del análisis (B1, S6, B6, B4, B3). En la quinta fase, se observó que se cumplió con la adecuacidad de la muestra $(K M O=.80$, $\left.\chi^{2}(171)=1,646.84\right)$. Los reactivos se agruparon en seis factores que explican el $69.41 \%$ de la varianza. Además, se observaron reactivos con cargas negativas (A5, A6, T5, T4, A1, A2) y que compartían carga factorial (E5), los cuales fueron eliminados.

En la sexta fase, la adecuacidad de la muestra se mantuvo de forma aceptable $\left(K M O=.76, \chi^{2}(66)=\right.$ 828.05). En esta ocasión, los reactivos se agruparon en cuatro factores que explican un $69.37 \%$ de la varianza. No se tuvieron que eliminar reactivos, por lo que la estructura factorial final quedó con cuatro factores. Los reactivos por factor fueron los siguientes: Factor 1 (S4, A4, B2, T1); Factor 2 (L3, L4, L5); Factor 3 (E1, E3); y Factor 4 (S1, S2, B5). Los reactivos del factor 1 contienen información relacionada a las prácticas organizacionales de bienestar en el trabajo. Los reactivos del factor dos van dirigidos a los logros profesionales. Los reactivos del factor tres expresan ideas de dificultad en el entorno social en el trabajo. Finalmente, los reactivos del factor cuatro van dirigidos a la salud y seguridad en el trabajo.

Se realizó un análisis de correlación entre los factores y se encontraron relaciones significativas que fluctuaron entre correlaciones bajas a moderadas bajas. La relación entre el Factor 1 y el Factor 2 fue positiva baja (Champion, 1981) y estadísticamente significativa. La relación entre el Factor 1 y el Factor 3 fue positiva baja y estadísticamente significativa de igual forma. En cuanto a la relación entre el Factor 1 y el Factor 4 se pudo observar una relación negativa 
moderada baja y estadísticamente significativa. Por otro lado, el Factor 2 y el Factor 3 se relacionaron de forma positiva baja y significativa. Sin embargo, la relación entre el Factor 2 y el Factor 4 fue negativa baja y significativa. Finalmente, se pudo observar que no hay una relación significativa entre el Factor 3 y el Factor 4. En la Tabla 2 se presentan las cargas factoriales y los índices de discriminación para cada reactivo, mientras que en la Tabla 3 se presentan las correlaciones entre los factores.

Tabla 2

Cargas Factoriales e Índices de Discriminación Versión Final

\begin{tabular}{|c|c|c|c|c|c|}
\hline & 1 & 2 & 3 & 4 & $r_{b i s}$ \\
\hline $\begin{array}{l}\text { S4. Mi trabajo me brinda dife- } \\
\text { rentes recursos, charlas y méto- } \\
\text { dos para promover estilos de } \\
\text { vida saludables. }\end{array}$ & .85 & & & & .68 \\
\hline $\begin{array}{l}\text { A4. La compañía se preocupa } \\
\text { por brindarme programas que } \\
\text { promuevan una alimentación } \\
\text { balanceada. }\end{array}$ & .71 & & & & .65 \\
\hline $\begin{array}{l}\text { B2. Mi compañía realiza activi- } \\
\text { dades recreativas que fomenten } \\
\text { la unión familiar. } \\
\text { T1. La organización para la que }\end{array}$ & .68 & & & & .60 \\
\hline $\begin{array}{l}\text { trabajo fomenta un proceso in- } \\
\text { formado sobre las prácticas del } \\
\text { trabajo remoto. }\end{array}$ & .43 & & & & .52 \\
\hline $\begin{array}{l}\text { L3. Me siento satisfecho con los } \\
\text { logros profesionales obtenidos. }\end{array}$ & & .83 & & & .70 \\
\hline $\begin{array}{l}\text { L4. He alcanzado logros signifi- } \\
\text { cativos a nivel profesional. }\end{array}$ & & .82 & & & .69 \\
\hline $\begin{array}{l}\text { L5. Siento que he realizado } \\
\text { aportaciones a mi carrera profe- } \\
\text { sional a través de mis experien- } \\
\text { cias laborales. }\end{array}$ & & .67 & & & .61 \\
\hline $\begin{array}{l}\text { E1. Tengo dificultad para rela- } \\
\text { cionarme con otras personas co- } \\
\text { tidianamente. }\end{array}$ & & & .78 & & .59 \\
\hline $\begin{array}{l}\text { E3. He tenido dificultad para } \\
\text { realizar actividades en mi } \\
\text { tiempo libre. }\end{array}$ & & & .77 & & .59 \\
\hline $\begin{array}{l}\text { S1. Los cambios organizaciones } \\
\text { o estructurales realizados en mi } \\
\text { lugar de trabajo me hacen sentir } \\
\text { seguro(a). }\end{array}$ & & & & .76 & .60 \\
\hline $\begin{array}{l}\text { S2. Conozco los riesgos que es- } \\
\text { tán relacionados a mi trabajo } \\
\text { que pueden afectar mi salud. }\end{array}$ & & & & .64 & .47 \\
\hline $\begin{array}{l}\text { B5. Mi trabajo promueve un } \\
\text { buen ambiente laboral basado } \\
\text { en la comunicación, respeto y } \\
\text { empatía. }\end{array}$ & & & & .54 & .53 \\
\hline Varianza Explicada (\%) & 31.76 & 15.42 & 12.92 & 9.27 & \\
\hline Valor Eigen & 3.81 & 1.85 & 1.55 & 1.11 & \\
\hline
\end{tabular}

\section{Análisis de Confiabilidad}

Se examinó el índice de discriminación de los reactivos que comprenden cada uno de los factores y se pudo observar que todos cumplieron adecuadamente con valores igual o mayores de .30. Para el Factor 1, el índice de discriminación fluctuó entre .52 a .68 . El Factor 2 tuvo valores que fluctuaron entre .61 a .70, mientras que para el Factor 3 fueron de .59, y para el Factor 4 fluctuaron entre .47 a .60. En cuanto a la consistencia interna, los cuatro factores obtuvieron valores alfa de Cronbach's aceptables: Factor $1(\alpha=.80)$, Factor $2(\alpha=.81)$, Factor $3(\alpha=.74)$, y Factor $5(\alpha=.71)$.

\section{Análisis de Correlación}

Se realizó un análisis de correlación Spearman para conocer la relación entre la precariedad laboral y los grupos generacionales. La precariedad laboral $(\alpha=.72)$ está compuesta por los reactivos S3 y S6 de la escala original de calidad de vida, sin embargo, estos reactivos no pertenecen a la versión final de la escala de calidad de vida. En cuanto a los grupos generacionales, estos se midieron en las categorías de edad establecidas (21-39 años, 40-60 años, y 61-75 años). Ambas variables son de carácter ordinal por lo que el análisis de correlación Spearman es de mayor utilidad para conocer la relación entre ambas variables. El resultado obtenido demostró una relación negativa baja y estadísticamente significativa entre las variables, $r_{s}=-.15, p<.05$. Este resultado implica que a mayor edad de la brecha generacional menor precariedad laboral o viceversa.

En adición, se realizó un análisis de correlación Producto Momento de Pearson para estudiar la relación entre la Precariedad Laboral y los Factores de Calidad de Vida. Los resultados obtenidos mostraron una relación negativa moderada baja y significativa entre la precariedad laboral y el Factor 1 de Prácticas Organizacionales de Bienestar, $r=-.30, p<.001$. Asimismo, se observó una relación negativa moderada baja y significativa entre la precariedad laboral y el Factor 2 de Logros Profesionales, $r=-.26, p<.001$. De igual forma, se pudo observar una relación negativa moderada baja y significativa entre la precariedad laboral y el Factor 3 de Entorno Social, $r=-.48, p<.001$. Por otro lado, se observó una relación positiva baja y significativa entre la precariedad laboral y el Factor 4 de Salud y Seguridad, $r=.17, p<.05$. 
Tabla 3

Correlación entre Factores

\begin{tabular}{|c|c|c|c|c|}
\hline & 1 & 2 & 3 & 4 \\
\hline F1: Prácticas Org. de Bienestar & $(.80)$ & & & \\
\hline F2: Logros Profesionales & $.25^{* *}$ & $(.81)$ & & \\
\hline F3: Entorno Social & $.24^{* *}$ & $.14^{*}$ & $(.74)$ & \\
\hline F4: Salud y Seguridad & $-.48^{* *}$ & $-.24^{* *}$ & -.07 & $(.71)$ \\
\hline
\end{tabular}

\section{Propiedades Psicométricas}

Para estudiar la estructura factorial del instrumento de calidad de vida desarrollado se realizó un análisis de factores exploratorio. En el análisis se incluyeron todos los reactivos del instrumento (34 reactivos). El análisis pasó por siete fases en total. En la primera fase se cumplió con la adecuacidad de la muestra $\left(K M O=.85, \chi^{2}(561)=3,645.60\right)$. Se detectaron ocho factores que explicaban el $65.59 \%$ de la varianza. Sin embargo, se tuvieron que eliminar reactivos porque compartían carga factorial (E6, T2, S3, T3, L2).

En la segunda fase se observó que se mantuvo la adecuacidad de la muestra $\left(K M O=.84, \chi^{2}(406)=\right.$ $2,835.54)$. Se mantuvieron de igual forma ocho factores explicando el $67.56 \%$ de la varianza. De forma similar a la fase uno, se eliminó un reactivo porque compartía carga factorial con otro factor (L1). En la tercera fase, se mantuvo la adecuacidad de la muestra $\left(K M O=.84, \chi^{2}(378)=2,733.25\right)$. En esta ocasión se observaron siete factores que explicaban un $65.07 \%$ de la varianza, y se eliminaron dos reactivos que compartían carga factorial con otros factores (S5, A3) y dos reactivos que no cumplieron con el mínimo de carga factorial (E4, E2).

En la cuarta fase, la adecuacidad de la muestra mantuvo sus valores dentro de lo esperado $(K M O=$ $\left..82, \chi^{2}(276)=2,165.34\right)$. En esta fase se observaron siete factores que explicaban un $68.31 \%$ de la varianza. Como se observaron reactivos con carga factorial negativa se procedieron a eliminar del análisis (B1, S6, B6, B4, B3). En la quinta fase, se observó que se cumplió con la adecuacidad de la muestra $(K M O=.80$, $\left.\chi^{2}(171)=1,646.84\right)$. Los reactivos se agruparon en seis factores que explican el $69.41 \%$ de la varianza. Además, se observaron reactivos con cargas negativas (A5, A6, T5, T4, A1, A2) y que compartían carga factorial (E5), los cuales fueron eliminados.
En la sexta fase, la adecuacidad de la muestra se mantuvo de forma aceptable $\left(K M O=.76, \chi^{2}(66)=\right.$ 828.05). En esta ocasión, los reactivos se agruparon en cuatro factores que explican un $69.37 \%$ de la varianza. No se tuvieron que eliminar reactivos, por lo que la estructura factorial final quedó con cuatro factores. Los reactivos por factor fueron los siguientes: Factor 1 (S4, A4, B2, T1); Factor 2 (L3, L4, L5); Factor 3 (E1, E3); y Factor 4 (S1, S2, B5). Los reactivos del factor 1 contienen información relacionada a las prácticas organizacionales de bienestar en el trabajo. Los reactivos del factor dos van dirigidos a los logros profesionales. Los reactivos del factor tres expresan ideas de dificultad en el entorno social en el trabajo. Finalmente, los reactivos del factor cuatro van dirigidos a la salud y seguridad en el trabajo.

Se realizó un análisis de correlación entre los factores y se encontraron relaciones significativas que fluctuaron entre correlaciones bajas a moderadas bajas. La relación entre el Factor 1 y el Factor 2 fue positiva baja (Champion, 1981) y estadísticamente significativa. La relación entre el Factor 1 y el Factor 3 fue positiva baja y estadísticamente significativa de igual forma. En cuanto a la relación entre el Factor 1 y el Factor 4 se pudo observar una relación negativa moderada baja y estadísticamente significativa. Por otro lado, el Factor 2 y el Factor 3 se relacionaron de forma positiva baja y significativa. Sin embargo, la relación entre el Factor 2 y el Factor 4 fue negativa baja y significativa. Finalmente, se pudo observar que no hay una relación significativa entre el Factor 3 y el Factor 4. En la Tabla 2 se presentan las cargas factoriales y los índices de discriminación para cada reactivo, mientras que en la Tabla 3 se presentan las correlaciones entre los factores.

\section{DISCUSIÓN}

El propósito de esta investigación fue presentar cómo en grupos generacionales se ve afectada su calidad de vida por la precariedad laboral durante el trabajo remoto. Así como, construir una escala de Calidad de Vida Laboral para evaluar diferentes aspectos tales como, bienestar, salud, alimentación balanceada, entorno social y cultural, logros profesionales y trabajo remoto. Por tal razón, durante el período de primavera 2021, en la investigación se recolectaron los datos por medio de la plataforma de PsychData utilizando las redes sociales como espacio para 
compartir el enlace. Hace varios años se ha estado experimentado una inestabilidad en el ámbito laboral y esta ha incrementado tras la actual pandemia del COVID-19, lo que ha intensificado la alta tasa de desempleo a nivel mundial (OIT, 2020).

En este estudio se establecieron tres preguntas de investigación, uno sobre el efecto de la precariedad laboral en la calidad de vida según las brechas generacionales. El rol de la precariedad laboral ante la calidad de vida. La escala de percepción de calidad de vida laboral durante el trabajo remoto de los empleados en Puerto Rico cumple con las propiedades psicométricas adecuadas para ser utilizada en investigaciones. A su vez, los datos recopilados en la presente investigación establecen que existe una relación entre las personas que indicaron sentir satisfacción laboral y las actuales condiciones de trabajo que su patrono les ofrece, por lo que indican contar con calidad de vida laboral y más al integrar el trabajo remoto como un nuevo estilo de vida laboral. Asimismo, González (2018) lo puntualiza en su investigación sobre: Nivel de satisfacción laboral en el rol docente y su relación con el síndrome de desgaste profesional en los maestros de colegios privados; este establece que, las condiciones de trabajo destacan como principales fuentes de insatisfacción laboral en el empleo.

Referente a la precariedad laboral y la calidad de vida en los grupos generacionales, se encontró que las diferencias radican entre los grupos de edad de 21-39 (Generación "Millenials") con el grupo de 61-75 años (Generación "Baby Boomers"). Las personas con edades entre 61-75 años obtuvieron puntuaciones significativamente mayores. Respecto al rol de la precariedad laboral ante la calidad vida, luego de los resultados obtenidos referente a la premisa que se les realizó a los participantes sobre si consideran que cuentan con calidad de vida laboral a lo que la mayoría contestó que sí. La calidad de vida laboral fundamentalmente se basa en una percepción que expresan los empleados de una organización sobre su grado de satisfacción o insatisfacción con respecto a su ámbito laboral, Cruz (2018). En cuanto a esto, es importante mencionar que las diferencias entre las generaciones causan cambios significativos con relación a las actitudes, los pensamientos y los comportamientos que una persona presente en su carrera profesional, así como su interés por este (Del Valle et al., 2019).

\section{Fortalezas y Limitaciones}

Una fortaleza de este estudio es el acceso virtual para publicar y que les brindó una accesibilidad segura a las personas para participar sin tener que exponer su salud. Además, el acceso virtual facilitó la recopilación de la muestra en un tiempo determinado. Dentro de las fortalezas es importante señalar que $87 \%$ de los participantes indicaron contar con calidad de vida. Otra fortaleza de esta investigación es el poder continuar realizando estudios sobre la misma utilizando la escala creada, atemperando las realidades laborales a los grupos generacionales y desarrollar mejores estrategias que incrementen las oportunidades de empleo integrando el trabajo remoto. De esta forma, aportar a la calidad de vida laboral de los trabajadores de este país. Cabe mencionar que, otra fortaleza de este estudio es que nos brinda una visión clara del sentir de los grupos generacionales o dimensiones con respecto a cuál se muestra más afectado por las variables de estudio. Por otra parte, en este estudio se encontraron tanto fortalezas como limitaciones.

Una de las de las limitaciones de este estudio fue la pandemia del COVID-19, ya que la muestra solo se recopiló únicamente de forma virtual para salvaguardar la seguridad de los participantes. Además, el tiempo limitado para completar la recopila-ción de los datos, ya que las personas estaban en un proceso de adapatación al trabajo remoto. Otra limitación encontrada fue que no se auscultó por completo sobre los beneficios entre estos los marginales que les ofrece la compañía a los empleados y que son vitales, ya que éstos se clasifican como las condiciones de trabajos y les proveen al empleado una calidad de vida laboral adecuada. A parte, al ser una muestra pequeña no representa el total de la población en Puerto Rico, por ende, se recomiendo continuar realizando investigaciones sobre el tema para brindar información concreta.

\section{Recomendaciones para Estudios Futuros}

Se recomienda continuar realizando investigaciones sobre el tema de la precariedad laboral y sus diferentes variables de interés. La precariedad laboral a incrementado a causa de la actual pandemia del COVID-19 que afecta a Puerto Rico y al mundo. Asimismo, lo puntualiza el (Departamento del Trabajo, 2021), en su encuesta de Grupo Trabajador donde 
establece que parte de los efectos de la actual pandemia del COVID-19, la cifra de desempleo señala que el 30.0\% de las personas desempleadas en abril 2021 bajo la categoría que agrupa principalmente a las personas desempleadas por razón del COVID-19. Es importante enfatizar que las investigaciones futuras deben estar enfocadas en ayudar a superar las limitaciones anteriormente presentadas. Es vital auscultar aspectos tales como: considerar el impacto que causa la precariedad laboral y cómo se ve afectada la familia.

Por último, en las investigaciones futuras sobre dicho tema se debe expandir a otros países, ya que el trabajo remoto fue una medida que las organizaciones optaron para continuar brindando un servicio a sus clientes, ya que la situación de la pandemia afectó a todos y no solo a Puerto Rico. De esta forma, permitiría obtener resultados más concretos y amplios sobre cómo se vio afectada la calidad de vida por generación durante el reto de trabajar remoto y cómo la precariedad laboral incrementó esto. Además, otra variable que se debe estudiar o incluir es el estrés para validar cómo este afecta la población y en específico a las generaciones. Asimismo, la información recopilada en esta investigación sea utilizada para estudios futuros, así ampliar las organizaciones de estudios para diversificar los resultados. Por último, utilizar el instrumento para la recopilación de los datos en las futuras investigaciones.

\section{CONCLUSIÓN}

En Puerto Rico se conocía que el tema de la precariedad laboral estaba afectando las oportunidades laborales, pero este fue incrementando más durante la pandemia del COVID-19. Así lo confirma (Venes, 2020), en su artículo sobre la pérdida de 50,000 empleos por la pandemia. A su vez, explica que la pandemia paralizó la actividad de construcción al mismo tiempo los fondos de CDBG DR los detuvieron. Añadiendo a su planteamiento que el estipendio de desempleo le estaba subiendo la tarifa por hora a las personas, esto significaba uno más algo de los que normalmente fluctúa. Aunque la mayoría de las organizaciones se adaptaron al cambio que provocó dicha pandemia y el recurrir al trabajo remoto para continuar brindando servicio a sus clientes, de igual forma, para salvaguardar la seguridad de sus empleados. Asimismo, muchas organizaciones se vieron obligadas a cerrar operaciones definitivamente por no contar con los recursos adecuados para acogerse al cambio radical. Esto provocó que la calidad de vida laboral de los individuos se viera afectada por el cambio de adaptarse a un método poco convencional, haciendo referencia al trabajo remoto y otros por las oportunidades limitadas de empleo, sino que también la vida en familia y la independencia de los mismos.

En este estudio sobre la relación entre la precariedad laboral y la calidad de vida en Puerto Rico no fue cónsona con las investigaciones previamente realizadas. Los datos recopilados en la investigación muestran una relación entre Precariedad Laboral y Calidad de Vida como una negativa moderada baja. De igual forma, se obtuvo una significancia entre la precariedad laboral y la dimensión de bienestar, logros profesionales, y entorno social. Solo hubo una relación positiva baja significativa entre la precariedad laboral y la salud. El análisis de los datos de este estudio muestra reflejó un resultado completamente inesperado ya que, según en la revisión de literatura de investigaciones previas, los factores anteriormente mencionados influyeron directamente en la satisfacción y calidad de vida laboral que espera un empleo en su ámbito laboral (González, 2018).

Financiamiento: La presente investigación no fue financiada por alguna entidad ni patrocinador.

Conflicto de Intereses: No existen conflictos de intereses de parte de los autores de la investigación.

Aprobación de la Junta Institucional para la Protección de Seres Humanos en la Investigación: IRB de la Universidad Albizu.

Consentimiento o Asentimiento Informado: Todo participante completó un consentimiento informado.

Proceso de Revisión: Este estudio ha sido revisado por pares externos en modalidad de doble ciego.

\section{REFERENCIAS}

Ato, M., López, J. J., \& Benavente, A. (2013). Un sistema de clasificación de los diseños de investigación en psicología. Anales de Psicología, 29(3), 1038-1059.

Benítez, I. (2016). La evaluación de la calidad de vida: Retos metodológicos presentes y futuros. Papeles del Psicólogo, 37(1), 69-73. http://www.papelesdelpsicologo.es/pdf/2664.pdf

Castro, P., Cruz, E., Hernández, J., Vargas, R., Luis, K., Gatica, L., \& Tepal, I. (2018). Una perspectiva de la calidad de vida laboral. Revista Iberoamericana de Ciencias, 5(6), 118-128. 
Coduti, P. S., Belén-Gattás, Y., Sarmiento, S. L., \& Schmid, R. A. (2013). Enfermedades laborales: Cómo afectan el entorno organizacional [Tesis de licenciatura, Universidad Nacional de Cuyo]. Biblioteca Digital UNCUYO. https://bdigital.uncu.edu.ar/objetos_digitales/5215/codutitesisenfermedadeslaborales.pdf

Cruz, J. E. (2018). La calidad de vida laboral y el estudio del recurso humano: Una reflexión sobre su relación con las variables organizacionales. Pensamiento \& Gestión, 45(1), 58-81. https://doi.org/10.14482/pege.45.10617

Custodio, J., Murawski, B., Elizathe, L., \& Rutsztein, G. (2017). Calidad de vida relacionada con la salud: Análisis factorial exploratorio del RAND -26 en mujeres de Buenos Aires. Revista Argentina de Ciencias del Comportamiento, 9(2), 34-49.

Del Valle, A., Martínez-Lugo, M., \& Rodríguez-Montalbán, R. (2019). Las generaciones en el trabajo en Puerto Rico: Una mirada desde la psicología industrial-organizacional y las etapas del desarrollo. Revista Salud y Conducta Humana, 6(1), 24-38.

Departamento del Trabajo y Recursos Humanos de Puerto Rico. (2021). Empleo y desempleo en Puerto Rico: Encuesta de Grupo Trabajador.

Díaz, C., López, M., \& Roncallo, L. (2017). Entendiendo las generaciones: Una revisión del concepto, clasificación y características distintivas de los baby boomers, $\mathrm{X}$ y millennials. Revista Clío América, 11(22), 188-204. https://doi.org/10.21676/23897848.2440

Díaz, R. (2019). Sentidos subjetivos de la precariedad en el empleo: Un estudio de caso. Revista Puertorriqueña de Psicología, 30(2), 342-356.

Fernández, J. (2017). NTIC y riesgos psicosociales en el trabajo: Estado de situación y propuestas de mejora. SAGGI, 2(2017), 69101. https://doi.org/10.14276/2531-4289.1278

García, T. (2018). Implicaciones psicosociales de la precariedad laboral en Puerto Rico. Psicologías, 1(2017), 54-64.

González, M. (2018). Nivel de satisfacción laboral en el rol docente y su relación con el síndrome de desgaste profesional en los maestros de colegios privados (Disertación doctoral, Universidad Metropolitana). Biblioteca Universidad Ana G. Méndez. https://documento.uagm.edu/cupey/biblioteca/biblioteca_tesisedudoc_gonzalezsanchezm2018.pdf

Hernández, R., Fernández, C., \& Baptista, P. (2017). Metodología de la investigación (6ta ed.). McGrawHill.

Marrero, J., \& García, T. (2018). Precariedad laboral: Aproximaciones teóricas e histórico sociales en el contexto de Puerto Rico. Psicología desde el Caribe, 35(Extra 0), 63-79.

Martínez, L. (2017). La precariedad laboral, manifestación de la mala calidad de vida. FLACSO. https://www.flacso.edu.ec/portal/files/docs/precariedad2.pdf

Montiel, M. (2020). Análisis de los grupos generacionales. ConCiencia Boletín Científico de la Escuela Preparatoria No.3, 7(14), 2427. https://repository.uaeh.edu.mx/revistas/index.php/prepa3/article/view/6111

Moreno, A. \& Camino, L. (2019). La precariedad laboral repercute en la salud de los jóvenes. Nueva Tribuna. https://www.nuevatribuna.es/articulo/economia-social/precariedad-laboral-repercute-salud-jovenes/20190415135538161916.html

Organización Internacional del Trabajo. (2020). COVID-19 deja un rastro de alta desocupación, inactividad y precariedad en el empleo en América Latina y el Caribe. https://www.ilo.org/americas/salade-prensa/WCMS_764643/lang--es/index.htm

Peralta-Beltrán, A. R., Bilous, A., Flores-Ramos, C. R., \& BombónEscobar, C. F. (2020). El impacto del teletrabajo y la administración de empresas. RECIMUNDO, 4(1), 326-335. https://doi.org/10.26820/recimundo/4.(1).enero.2020.326-335

Real Academia Española. (2020). Logro.

Romina, P. (2016). Calidad de vida [Monografía]. Universidad de Palermo.

Venes, P. (2020, agosto 28). Puerto Rico perdió cerca de 50,000 empleos por la pandemia. Noticel. https://www.noticel.com/economia/coronavirus/top-stories/20200828/puerto-rico-perdiocerca-de-50000-empleos-por-la-pandemia/ 